\title{
EFECTO DE LA HARINA DE PESCADO Y DEL AGUA VERDE CON DOMINANCIA DE Chlorella sp y Scenedesmus sp. EN LA PRODUCCIÓN EXPERIMENTAL DE Moina sp. (CLADÓCERA)
}

\author{
Wilson RODRÍGUEZ ${ }^{1}$, Fransisk SÁNCHEZ², Fernando ALCÁNTARA \\ 1 Universidad Nacional Agraria de la Selva. Tingo María. E. mail: watson312@hotmail.com \\ 2 Universidad Nacional Federico Villarreal. Lima. E. mail: Ni-pelg-you@hotmail.com \\ 3 Instituto de Investigaciones de la Amazonía Peruana. Programa de Investigación para el Uso y Conservación del Agua y \\ sus Recursos. Carretera Iquitos Nauta. Km. 4.5. Iquitos. Perú. E. mail: fab_0005@hotmail.com
}

\begin{abstract}
RESUMEN
Este estudio fue ejecutado en el Centro de Investigaciones de Quistococha del Instituto de Investigaciones de la Amazonia Peruana, situado en Loreto, Perú con la finalidad de determinar la influencia de la harina de pescado y del inóculo de Chlorella sp., y Scenedesmus sp., en la producción experimental de Moina sp., (Cladócera). Fueron utilizados cuatro tratamientos y cuatro repeticiones en un diseño completamente al azar. En los tratamientos fueron utilizados 1.0, 1.5, 2.0 y $2.5 \mathrm{~g} . \mathrm{L}^{-1}$ de harina de pescado, en adición a $0.2 \mathrm{~L}$ de agua verde con dominancia de Chlorella sp., y Scenedesmus sp. La producción promedio de cladóceros fue proporcional a los cuatro niveles de harina de pescado utilizados, según se indica: $37725 \pm 7090.48,11675 \pm$ $4474.73,492 \pm 284.50$ y $1642 \pm 1641.75$ org. $\mathrm{L}^{-1}$, respectivamente. El análisis de varianza y el test de Tukey al nivel de probabilidad de 0.05 mostraron diferencia significativa entre el tratamiento uno y los demás tratamientos $(\mathrm{p}<0.05)$. La mayor producción fue observada en el tratamiento con $1 \mathrm{gL}^{-1}$ de harina de pescado y que presentó un nivel de $\mathrm{pH}$ de 9.6. Los niveles de harina de pescado superiores a $1 \mathrm{gL}^{-1}$ determinaron bajos niveles de producción, presentando nivel de $\mathrm{pH}$ de $6.6 \pm 2.3$, lo cual afectó negativamente la producción final de cladóceros. La concentración de oxígeno disuelto fue de $2.3 \pm 1.4 \mathrm{mgL}^{-1}$ y la del pH fue de $6.6 \pm 2.3$ mientras que la temperatura fue de $22.8 \pm 3.8^{\circ} \mathrm{C}$.
\end{abstract}

PALABRAS CLAVE: Moina sp., Cladócera, cultivo, harina pescado.

\section{EFFECT OF FISH FLOUR AND THE GREEN WATER WITH DOMINANCE OF Chlorella sp. and Scenedesmus sp. IN THE EXPERIMENTAL PRODUCTION OF Moina sp. (CLADOCERA)}

\begin{abstract}
This study was carried out at the Quistococha Research Center of the Peruvian Amazon Research Institute located in Loreto, Peru, in order to determine the influence of fishmeal and the inoculum of Chlorella sp., and Scenedesmus sp., in the experimental production of Moina sp. Four treatments and four replications were used in a completely randomized design. In the treatments, we used 1.0, 1.5, 2.0 and $2.5 \mathrm{~g} . \mathrm{L}^{-1}$ of fish flour, in addition to $0.2 \mathrm{~L}$ of green water with dominance of Chlorella sp., and Scenedesmus sp. The average production of cladocerans was proportional to the use of four fish flour quantities as indicated: $37725 \pm$ $7090.48 ; 11675 \pm 4474.73 ; 492 \pm 284.50$ and $1642 \pm 1641.75$ org. $\mathrm{L}^{-1}$. The analysis of variance and the Tukey test at the probability level of 0.05 showed a significant difference between treatment one and the other three treatments $(\mathrm{p}<0.05)$. The highest production was observed in the treatment with $1 \mathrm{gL}^{-1}$ of fish flour and this showed a pH level of 9.6. The levels of fish flour higher than $1 \mathrm{gL}^{-1}$ determined low production and presented $\mathrm{pH}$ levels of $6.6 \pm 2.3$ affecting negatively the final production of cladocerans. The concentration for the treatments was $2.3 \pm 1.4 \mathrm{mgL}^{-1}$ of dissolved oxygen, $6.6 \pm 2.3 \mathrm{of} \mathrm{pH}$ and $22.8 \pm 3.8^{\circ} \mathrm{C}$ of temperature.
\end{abstract}

KEYWORDS: Moina, Cladocera culture, fish flour 


\section{INTRODUCCIÓN}

Moina sp. es un microcrustaceo filtrador no selectivo de importancia relevante, tiene una talla de $1.0 \mathrm{~mm}$ al estado adulto y de $0.4 \mathrm{~mm}$ en los neonatos (Prieto et al., 2006), presenta un contenido proteico alrededor de $50 \%$ y de grasa del 20 al $27 \%$ en los adultos y del 4 al $6 \%$ en los juveniles (Jiménez et al., 2003). El contenido de lípidos de la Moina es de gran importancia para sus propias necesidades metabólicas, como para las especies que la consumen (Goulden et al., 1988, citado por Romero, 2009).

Los cladóceros han sido cultivados utilizando Chlorella y Ankistrodesmus (Prieto, 2001); Ankistrodesmus y Saccharomyces cereviseae (Prieto, 2006); Scenedesmus quadricauda y Ankistrodesmus gracilis (Macedo \& Pinto-Coelho, 2001) y otros, como levadura Z-plus y micro algas (Jiménez et al., 2003). La Moina se alimenta de varios grupos de bacterias, fitoplancton y detritus y sus poblaciones crecen más rápidamente en presencia de adecuadas cantidades de bacterias y fitoplancton. (Rottmann et al., 2011). La disponibilidad de alimento de calidad y en la cantidad necesaria, juega un rol determinante en el crecimiento y la sobrevivencia en la fase de larvicultura y alevinaje de peces en condiciones de cultivo. En estas fases se usa, frecuentemente, nauplios de Artemia que, son caros y no siempre están disponibles, con la desventaja adicional como lo reportaron Rottmann et al. (2011) de la escasa sobrevivencia de los nauplios en agua dulce. Ocampo et al. (2010) ejecutaron el cultivo de Daphnia magna alimentándola con probióticos y dietas a base de Saccharomyces cereviseae y un medio enriquecido con ácidos grasos $(\mathrm{n}-6)$ provenientes de harina avena-soya concluyendo que los Cladóceros presentan adaptación favorable a las condiciones de manejo para la producción de biomasa útil como alimento vivo en acuicultura.

En este contexto, es necesario buscar métodos alternativos, simples, de bajo costo y accesibles de producción de alimento de calidad, como los cladóceros y, entre ellos, de Moina sp., para su utilización en la fase de larvicultura de peces y otros organismos utilizados en acuicultura. Este estudio fue ejecutado con la finalidad de determinar la influencia de la harina de pescado como fuente de nutrientes en la producción experimental de Moina sp.

\section{MATERIAL Y MÉTODOS}

El experimento fue ejecutado en el Centro de Investigaciones de Quistococha del Instituto de
Investigaciones de la Amazonía Peruana en el 2011, en dos etapas: En la primera, fueron capturadas dos Moina adultas en el módulo de producción de alimento vivo con fines de larvicultura y posteriormente aisladas en un beaker de $200 \mathrm{ml}$, a razón de un individuo por cada unidad experimental. Como alimento fue ofrecido filtrados de micro algas con dominancia de Chlorella sp., y Scenedesmus sp., procedentes del módulo de producción de alimento vivo del C.I Quistococha.

Al día siguiente de la separación de los adultos fue observada la sobrevivencia de una Moina y la producción de 20 neonatos. En la segunda etapa fueron sembrados dos neonatos en cada unidad experimental compuesta por bolsas de plástico de 10 L de capacidad instaladas con los tratamientos de harina de pescado como fuente de nutrientes, como se indica:

T1: 1 gramo de harina de pescado por litro de agua. T2: 1.5 gramos de harina de pescado por litro de agua.

T3: 2.0 gramos de harina de pescado por litro de agua.

T4: 2.5 gramos de harina de pescado por litro de agua.

Treinta horas antes de la siembra de los cladóceros, fueron llenadas las unidades experimentales con agua filtrada en una malla de 60 $\mu \mathrm{m}$, hasta un volumen de $9.8 \mathrm{~L}$ completando el volumen a $10 \mathrm{~L}$ con la adición de $0.2 \mathrm{~L}$ de agua verde con dominancia de Chlorella y Scenedesmus, filtrada en una malla de 33 micras.

Las unidades experimentales, fueron ubicadas en cuatro columnas y cuatro filas en un diseño completamente al azar bajo un área techada de $4 \times 4$ $\mathrm{m}$, con techo de plástico transparente para protegerlas de la lluvia y dejar pasar la radiación solar directa y difusa.

La distribución de los tratamientos y sus repeticiones fue determinada mediante el programa Random Sequence Generator, (www.random.org /sequences).

Fueron evaluadas las variables físicas y químicas de las unidades experimentales. El oxígeno disuelto y la temperatura fueron evaluados mediante un oxímetro marca YSI, Modelo 55-12 FT y el pH fue medido mediante un equipo digital marca Hanna.

La producción de cladóceros fue evaluada al cabo de 12 días, mediante el siguiente procedimiento: Se filtró el contenido de cada una de las unidades experimentales con una malla de 50 
micras, hasta alcanzar un volumen de $200 \mathrm{ml}$ que fue retenido en un beaker; luego con una micro pipeta Eppendorf de 500 micro litros se tomó 6 muestras de $0.5 \mathrm{ml}$ cada una, para realizar el conteo directo de los cladóceros, en una placa Petri, paralizándolos de antemano, con la ayuda de alcohol al $70 \%$ de acuerdo a Rottmann et al. (2011). La producción final fue estimada relacionando el número de cladóceros de la muestra con el volumen total del concentrado.

Los resultados fueron analizados utilizando la hoja de cálculo Excel y el Programa estadístico Statistical Package for the Social Sciences (SPSS) versión 18 determinando los estadísticos media, mínimo, máximo, desviación típica, varianza, coeficiente de correlación y test de Tukey.

\section{RESULTADOS Y DISCUSIÓN}

La producción promedio de cladóceros en los tratamientos fue $37725 \pm 7090.48 ; 11675 \pm 4474.73$; $492 \pm 284.50$ y $1642 \pm 1641.75$ org..$^{-1}$ (media \pm error típico de la media), inversamente proporcional a la concentración de harina de pescado $(\mathrm{T} 1>\mathrm{T} 2>\mathrm{T} 3>\mathrm{T} 4)$. Tabla 1. Figura 1. El análisis de varianza indicó diferencia significativa entre los tratamientos (Tabla 2) y el test de Tukey (Tabla 3) evidenció diferencia significativa entre el tratamiento uno y los demás tratamientos indicando que $1 \mathrm{~g}$ de harina de pescado por litro de agua utilizado en el tratamiento uno fue el más adecuado para la producción de los Cladóceros en las condiciones experimentales $(\mathrm{p}<0.05)$. Figura 1 .

Tabla 1. Producción de cladóceros utilizando harina de pescado como fuente de nutrientes (org. $\mathrm{L}^{-1}$ ).

\begin{tabular}{ccrrrrr}
\hline $\begin{array}{c}\text { Tratamientos } \\
\text { (H. pescado } \mathbf{~ g L}^{-1} \text { ) }\end{array}$ & N & Media & Desv. típ. & Mínimo & Máximo & $\begin{array}{c}\text { Error típ. } \\
\text { de la media }\end{array}$ \\
\hline 1.0 & 4 & 37725 & 14180.96 & 20900 & 50800 & 7090.48 \\
1.5 & 4 & 11675 & 8949.45 & 2733 & 20200 & 4474.73 \\
2.0 & 4 & 492 & 569.00 & 0 & 1033 & 284.50 \\
\hline 2.5 & 4 & 1642 & 3283.50 & 0 & 6567 & 1641.75 \\
\hline Total & 16 & 12883 & 17264.39 & 0 & 50800 & 4316.10 \\
\hline
\end{tabular}

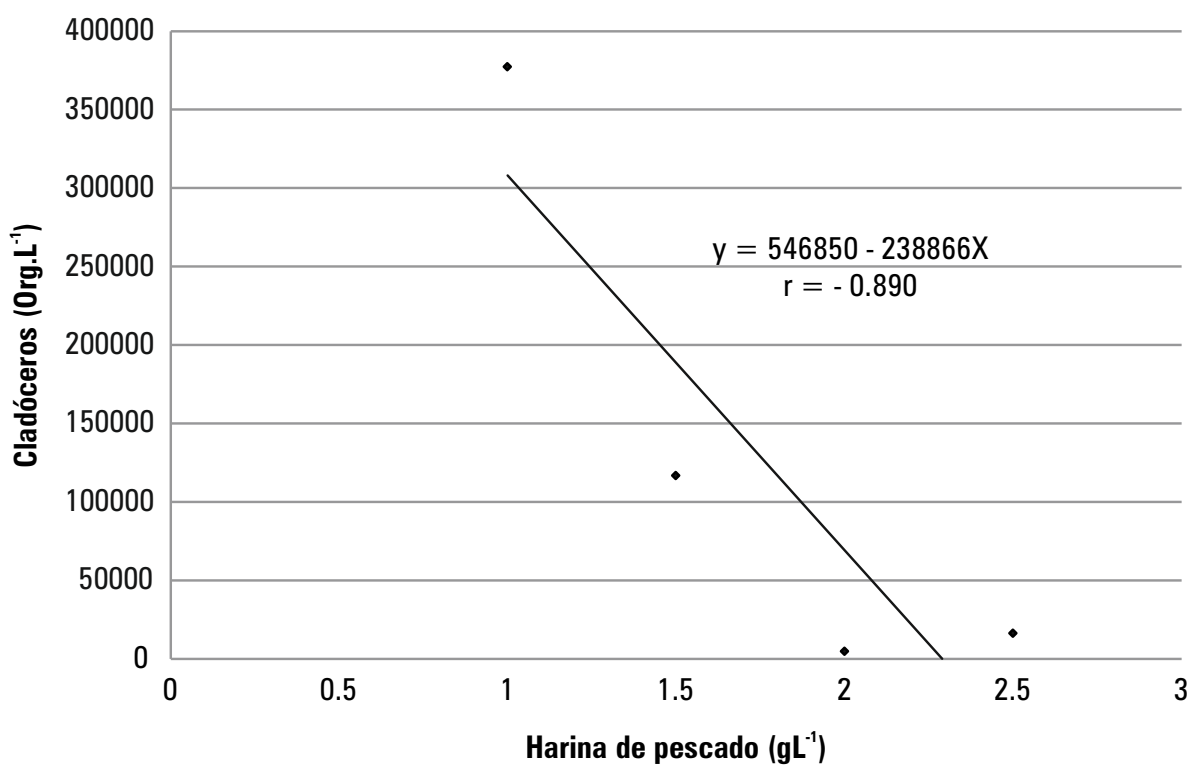

Figura 1. Producción de cladóceros por tratamiento. 
Tabla 2. Análisis de varianza.

\begin{tabular}{|c|c|c|c|c|c|c|c|}
\hline & & & $\begin{array}{l}\text { Suma de } \\
\text { cuadrados }\end{array}$ & gl & $\begin{array}{l}\text { Media } \\
\text { cuadrática }\end{array}$ & $\mathbf{F}$ & Sig. \\
\hline $\begin{array}{l}\text { Número de } \\
\text { Cladóceros }\end{array}$ & Inter-grupos & (Combinadas) & 3593996805.688 & 3 & 1197998935.229 & 16.394 & 0.000 \\
\hline \multirow[t]{2}{*}{ * Tratamientos } & Intra-grupos & & 876891961.750 & 12 & 73074330.146 & & \\
\hline & Total & & 4470888767.438 & 15 & & & \\
\hline
\end{tabular}

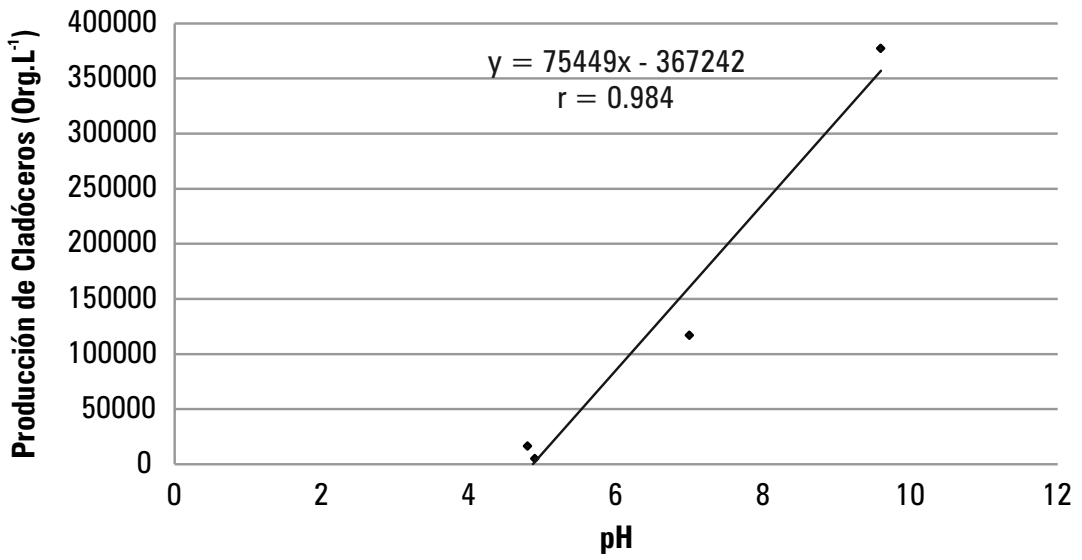

Figura 2. Producción de Cladóceros y niveles de pH en la utilización de harina de pescado como fuente de nutrientes.

Los niveles superiores a $1 \mathrm{~g}$ de harina de pescado presentaron menor producción de cladóceros, con una correlación negativa significativa $(\mathrm{y}=546850$ 238866X; $r=-0.8909$ ) entre los niveles de harina de pescado y la producción de cladóceros (Figura 1) debido aparentemente, a condiciones de calidad de agua inadecuadas para el desarrollo de los organismos por acidificación del medio de cultivo (Figura 2 y 3 ) y probablemente niveles inadecuados de nitrito derivado de la harina de pescado utilizada como fuente de nutrientes.

La estrategia de cultivo de Moina sp., utilizando diversos niveles de harina de pescado y filtrados de plancton con dominancia de Chlorella y Scenedesmus, efectuada en este experimento, fue eficaz en el tratamiento uno (T1) porque permitió la sobrevivencia de los neonatos que alcanzaron el estado adulto y produjeron sus propios descendientes. Aún cuando en el tratamiento dos (T2) con $1.5 \mathrm{gL}^{-1}$ de harina de pescado fue observada sobrevivencia y producción de cladóceros, los niveles de producción fueron bajos en relación a los niveles alcanzados en el tratamiento uno, con $1 \mathrm{gL}^{-1}$ de harina de pescado. En este sentido, nuestros resultados nos permiten identificar la necesidad de continuar las investigaciones en esta estrategia de producción en orden a determinar el nivel óptimo de carga de harina de pescado que permita la mayor producción posible de cladóceros. No hemos registrado antecedentes de utilización de harina de pescado como fuente de nutrientes en el cultivo de Cladóceros por lo cual es difícil establecer comparaciones del método utilizado en este trabajo. Sin embargo, Martínez (1999) y Prieto (2001) reportaron el uso de Chlorella y Ankistrodesmus en el cultivo de Moinodaphnia; Jiménez et al. (2003) utilizaron tres dietas: la micro alga Scenedesmus abundans la levadura Saccharomyces cereviseae de la marca Safinstant ${ }^{\circledR}$ y un alimento formulado para zooplancton de la marca comercial Z-plus ${ }^{\circledR}$; Romero (2009) reportó el uso del Scenedesmus como alimento de Moina; Núñez \& Hurtado (2005) utilizaron jugo de alfalfa y levadura en el cultivo de Daphnia magna; Martínez (2000) reportó la 
conducta alimentaria de Moina micrura, Ceriodaphnia dubia y Daphnia ambigua (Cladócera) frente a las micro algas Chlorella sp., y Oocystis sp., (Chlorophyceae); Rodríguez-Estrada et al. (2003) reportaron que Moina micrura fue cultivada empleando como alimento las micro algas clorofíceas Ankistrodesmus falcatus y Scenedesmus incrassatulus; Prieto et al. (2006) utilizaron Ankistrodesmus sp., y Ankistrodesmus + Saccharomyces sereviseae (levadura de panificación); Ocampo et al. (2010) realizaron el cultivo experimental del cladócero Daphnia magna utilizando probióticos como alimento; Rottmann et al. (2011) reportaron que en Singapur la Moina micrura crece en estanques fertilizados con excretas de gallina o menos frecuentemente, con estiércol de cerdo; Damle \& Chari (2011) evaluaron la influencia de las excretas de vacuno, cabra y pollo en el rendimiento de Daphnia sp.; Ramírez-Merlano et al. (2013) reportaron que los cladóceros, en especial el género Moina fueron alimentados con una suspensión de micro algas Scenedesmus sp.

Prieto \& Atencio (2008) reportaron que la larvicultura de peces neo tropicales altriciales presenta limitaciones para el manejo de la primera alimentación dado el pobre desarrollo del tracto digestivo y capacidad natatoria, por lo tanto, la alimentación de la larva debe considerar el tamaño, densidad y calidad de la presa ofrecida. En este sentido, consideramos que el trabajo realizado representa una alternativa simple de producción de organismos de gran calidad nutritiva y de tamaño adecuado para la alimentación de las larvas de peces y otros organismos en cultivo.

Tabla 3. Test de Tukey

\begin{tabular}{ccrcccc}
\hline \multirow{2}{*}{ (I) Tratamientos } & $\begin{array}{c}\text { Diferencia } \\
\text { de medias (I-J) }\end{array}$ & Error típico & Sig. & \multicolumn{2}{c}{ Intervalo de confianza al 95\% } \\
\cline { 5 - 7 } & & & & Límite inferior & Límite superior \\
\hline 1.0 & 1.5 & $26050,00000^{*}$ & 6044.59801 & 0.005 & 8104 & 43996 \\
& 2.0 & $37233,50000^{*}$ & 6044.59801 & 0.000 & 19288 & 55179 \\
\hline 1.5 & 2.5 & $36083,25000^{*}$ & 6044.59801 & 0.000 & 18137 & 54029 \\
\hline & 1.0 & $-26050,00000^{*}$ & 6044.59801 & 0.005 & -43996 & -8104 \\
& 2.0 & 11184 & 6044.59801 & 0.299 & -6762 & 29129 \\
\hline 2.0 & 2.5 & 10033 & 6044.59801 & 0.385 & -7913 & 27979 \\
\hline & 1.0 & $-37233,50000^{*}$ & 6044.59801 & 0.000 & -55179 & -19288 \\
& 1.5 & -11184 & 6044.59801 & 0.299 & -29129 & 6762 \\
\hline 2.5 & 2.5 & -1150 & 6044.59801 & 0.997 & -19096 & 16796 \\
\hline & 1.0 & $-36083,25000^{*}$ & 6044.59801 & 0.000 & -54029 & -18137 \\
& 1.5 & -10033 & 6044.59801 & 0.385 & -27979 & 7913 \\
\hline
\end{tabular}

* La diferencia de medias es significativa al nivel 0.05 .

Tabla 4. Calidad de agua en las unidades experimentales de producción de Cladóceros.

\begin{tabular}{lcccc}
\hline & Tratamiento & $\begin{array}{c}\text { 0xígeno disuelto } \\
(\mathbf{m g L}-\mathbf{1})\end{array}$ & $\mathbf{p H}$ & $\begin{array}{c}\text { Temperatura } \\
\left({ }^{\mathbf{}} \mathbf{C}\right)\end{array}$ \\
\hline $\mathrm{N}$ Válidos & 7 & 4 & 4 & 4 \\
$\quad$ Perdidos & 0 & 3 & 3 & 3 \\
Media & & 2.3 & 6.6 & 22.8 \\
Desv. típ. & & 1.4 & 2.3 & 3.8 \\
Rango & & 3.1 & 4.8 & 7.7 \\
Mínimo & & 1.3 & 4.8 & 19.2 \\
Máximo & & 4.4 & 9.6 & 26.9 \\
\hline
\end{tabular}




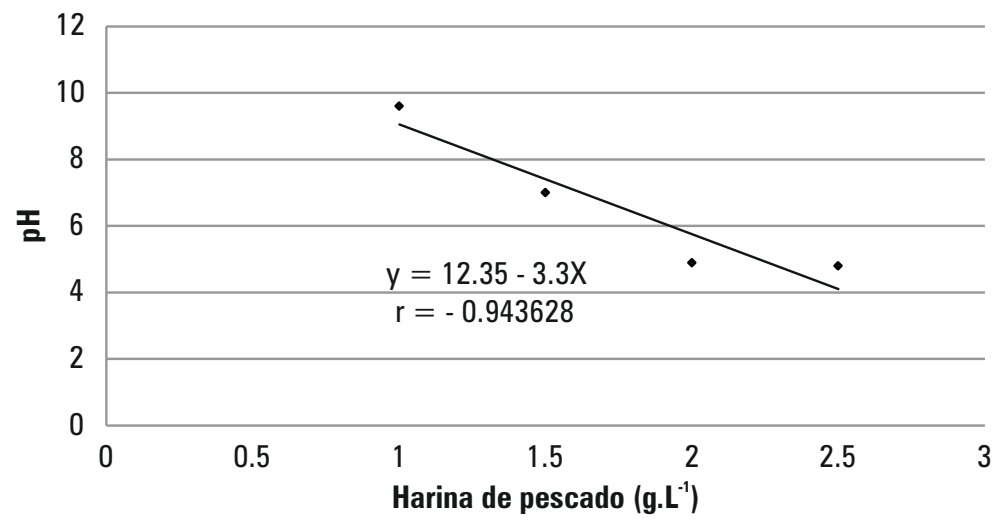

Figura 3. Niveles de pH alcanzados en la utilización de harina de pescado como fuente de nutrientes en la producción experimental de Cladóceros.

El método de cultivo constituye una innovación que puede contribuir en la producción de cladóceros en condiciones experimentales por cuanto la harina de pescado es un insumo relativamente barato, ampliamente disponible y puede proporcionar los nutrientes necesarios para la producción de micro algas alimenticias de los cladóceros como son Chlorella, Scenedesmus, Ankistrodesmus, Oocystis, proporcionando a la vez, pequeñas partículas de materia orgánica y bacterias que pueden ser aprovechadas directamente por los cladóceros.

La mayor producción de Cladóceros fue alcanzada en niveles de $\mathrm{pH}$ de 9.6 con una correlación positiva entre esta variable y la producción de Cladóceros $\left(\mathrm{R}^{2}=0.9686\right)$. Figura 2 . Los niveles de harina de pescado superiores a $1 \mathrm{gL}^{-1}$ utilizados, determinaron la acidificación del medio de cultivo hasta niveles de $\mathrm{pH}$ menores que 5 inadecuados para la productividad de los organismos planctónicos en general y en particular para la producción de Chlorella sp. y Scenedesmus sp., inoculados en el agua verde al inicio del experimento, con lo cual, fue observada una correlación negativa entre los niveles de harina de pescado y los niveles de $\mathrm{pH}\left(\mathrm{R}^{2}=0.8904\right)$, Figura 3 .

La concentración del oxígeno disuelto fue de 2.3 $\pm 1.4 \mathrm{mg} . \mathrm{L}^{-1}$, el pH fue de $6.6 \pm 2.3$ y la temperatura fue de $22.8 \pm 3.8^{\circ} \mathrm{C}$ que, en términos generales, determinaron condiciones adecuadas para el desarrollo de los cladóceros, en los diversos tratamientos, Tabla 4.

Scout et al. (2006) citado por Romero (2009) consideran que la Moina sp., se adapta a rangos amplios de temperatura, aguas con cierto grado de contaminación orgánica y concentraciones de oxígeno desde cero hasta la sobresaturación.

En este sentido, consideramos que, los niveles de temperatura y oxígeno disuelto han sido adecuados para la producción de cladóceros, pero es probable que, la acidificación del medio de cultivo, los sólidos en suspensión y sobre todo los niveles de nitrito derivados de la harina de pescado que no fueron determinados, hayan influido negativamente en el fitoplancton y en la producción de los cladóceros.

\section{BIBLIOGRAFÍA CITADA}

Damle, D.; Chari, M. 2011. Performance Evaluation of Different Animal Wastes on Culture of Daphnia sp. Journal of Fisheries and Aquatic Science, 6(1): 57-61.

Jiménez, D.; Rosas, J.; Velásquez, A.; Millán, J.; Cabrera, T. 2003. Crecimiento poblacional y algunos aspectos biológicos del cladócero Moina macrocopa (Straus, 1820) (Branchiopoda, Anomopoda), alimentado con tres dietas en tres salinidades diferentes. Ciencia, 11(1): 22-30.

Macedo, C.; Pinto-Coelho, R. 2001. Nutritional status response of Daphnia laevis and Moina micrura from a tropical reservoir to different algal diets: Scenedesmus quadricauda and Ankistrodesmus gracilis. Programa de Postgraduação em Ecología, conservação e Manejo da Vida Silvestre (ECMVS). Bras. J. Biol., 61(4): 555-562 
Martínez, G. 1999. Estrategias de alimentación de tres especies del zooplancton límnico (Cladócera) http://rchn.biologiachile.cl/pdfs /1999/4/Martinez_1999.pdf. Acceso: 28 de febrero de 2012.

Martínez, G. 2000. Conducta alimentaria de Daphnia ambigua Scourfield 1947, Moina micrura kurz 1874 y Ceriodaphnia dubia Richard 1895 (Cladócera) frente a un gradiente de concentración de alimento. http://www. scielo.cl/pdf/rchnat/v73n1/art06.pdf. Acceso: 7 de marzo del 2012.

Núñez, M.; Hurtado, J. 2005. Bioensayos de toxicidad aguda utilizando Daphnia magna Straus (Cladocera, Daphniidae) desarrollada en medio de cultivo modificado. Facultad de Ciencias Biológicas. UNMSM. Rev. peru. biol., 12(1): 165-170.

Ocampo, L., Botero, M., Restrepo, L., 2010. Evaluación del crecimiento de un cultivo de Daphnia magna alimentado con Saccharomyces cereviseae y un enriquecimiento con avena soya. Revista Colombiana de Ciencias Pecuarias, Vol. 23, No 1: 78-85.

Prieto, M. 2001. Aspectos reproductivos del cladócero Moinodaphnia sp., en condiciones de laboratorio.http://redalyc.uaemex.mx/pdf/693 /69360205.pdf. Acceso: 1 de febrero del 2012.

Prieto, M.; de la Cruz, L.; Morales, M. 2006. Cultivo experimental del Cladócero Moina sp., alimentado con Ankistrodesmus sp., y Saccharomyces cereviseae. Universidad de Córdoba. Rev. MVZ. Córdoba, 11(1): 705-714.
Prieto, M.; Atencio. 2008. Zooplancton en la larvicultura de peces neotropicales. Rev. MVZ. Córdoba, 13(2): 1415-1425.

Ramírez - Merlano, J.; Mira - López, T.; CruzCasallas, P. 2013. Efecto de la intensidad lumínica sobre la eficiencia reproductiva del cladócero Moina sp., bajo condiciones de laboratorio. Orinoquia, Universidad de Los Llanos Meta, Colombia. Orinoquia. Vol. 17. Núm. 2: 177-182.

Rodríguez-Estrada, J.; Villaseñor-Córdova, R.; Martínez-Jerónimo, F. 2003. Efecto de la temperatura y tipo de alimento en el cultivo de Moina micrura (Kurz, 1874) (Anomopoda: Moinidae) en condiciones de laboratorio. Hidrobiológica, 13 (3): 239-246.

Romero, T. 2009. Desarrollo de Moina sp en condiciones de laboratorio alimentada con micro algas cultivadas en residuales pesqueros. http://www.veterinaria.org/revistas/redvet/n 040409/040915. pdf, documento, Acceso: 9 de marzo de 2012.

Rottmann, R.; Scott, J.; Watson, C.; Yanong, R. 2011. Culture techniques of Moina: The ideal Daphnia for Feeding Freshwater Fish Fry. University of Florida. IFAS Station. CIR 1054: 07.

Recibido: 10 de Junio del 2016

Aceptado para publicación: 17 de Julio del 2016 
